\title{
The Relationship Between Learning Style, Self-Directed in Learning and Undergraduate Nursing Students' Academic Achievement in Tehran University of Medical Science (TUMS)
}

\author{
Addis Adera Gebru ${ }^{1, ~ *}$, Shahrazad Ghiyasvandian ${ }^{1}$, Nooroodin Mohammadi ${ }^{2}$ \\ ${ }^{1}$ School of Nursing and Midwifery, Tehran University of Medical Sciences, Department of Medical Surgical Nursing, Tehran, Iran \\ ${ }^{2}$ School of Nursing and Midwifery, Tehran University of Medical Sciences, Department of Critical Care Unit, Tehran, Iran
}

Email address:

addisaderagebru@gmail.com (A. A. Gebru)

To cite this article:

Addis Adera Gebru, Shahrazad Ghiyasvandian, Nooroodin Mohammadi. The Relationship Between Learning Style, Self-Directed in Learning and Undergraduate Nursing Students' Academic Achievement in Tehran University of Medical Science (TUMS). American Journal of Nursing Science. Vol. 4, No. 3, 2015, pp. 138-150. doi: 10.11648/j.ajns.20150403.24

\begin{abstract}
Background: Self-Directed in Learning has been emphasized nursing education settings based on many factors such as changing in learning teaching methods, nursing professions development, etc. Awareness and identification of the students learning and their academic achievement are important factors in nursing Course. Objective: The purpose of this study was to determine the relationship between self-directed in learning and undergraduate nursing students' academic achievement in Tehran University of Medical Sciences (TUMS), Tehran, Iran. Method: The cross sectional study with 232 sample size was conducted. The subjects of this study were 232 undergraduate nursing students from school grades of years attending to school of nursing and midwifery of Tehran University of Medical Sciences. Data was collected from students through an anonymous self-administrated questionnaire. The questionnaire was divided into three sections including (a) demographic profile (b) Fisher's Self-Directed in Learning Readiness questionnaire, (d) academic achievement. Data analysis was carried out by using the latest version of the statistical software package SPSS (Version-21). Descriptive and analytical statistical test were used to analyze the data. Results: A total of 232 participated in study of relationship between learning styles, Self-directed in learning and undergraduate nursing students' academic achievement. One hundred -Forty three participants were female (61.6\%), and 89 respondents were male (38.4\%); $60.8 \%$ were between 20 and 25 years, $33.6 \%$ were $<20$ years, and $5.6 \%$ were $>25$ years old. The most frequency learning style of students was AC (37.5\%).The majority of subjects (90.52\%) SDLR were in the level of Self-Control (Score $>150)$. The majority of subjects $(52.8 \%)$ their academic achievements were at level of Good (the Median score were between (13-16). There was no significant relationship between Learning styles and Academic Achievement ( $\mathrm{P}>$ 0.05).Conclusion: Not all students are self-directed, and this study suggests that mature students are more self-directed than that entering nurse education direct from high school. Nurses' educators need to assess the Learning style and preferences of their students in order to determine the appropriateness of Self-Directed in learning. It is important to acknowledge that SelfDirected in Learning is only one teaching method that can be used to meet the learning needs of all students.
\end{abstract}

Keywords: LS, Academic Achievement, Self-Directed Learning, Nursing Students', Iran, Undergraduate Degree

\section{Introduction}

Learning is the dynamic process of changes that it happens throughout a persons' life. Teaching learning contributes to the development of professional values, attitudes and behavior through a partnership of accountability between teacher and learner (1). Learning is described as the process whereby knowledge is created through the transformation of experience. Individuals use learning to adapt to and manage everyday situations, giving rise to different styles of learning. The concept of Learning styles(LSs) has received considerable attention in the empirical literature and many theories have been proposed in order to better understand the dynamic process of 
learning) $(2,, 3,4,5)$.Term of "LS refers to this view that different people learn information in different ways "and "refers to the concept that individuals differ in regard to what mode of instruction or study is most effective for them" (6).During the last two decades, the LS and learning reference of college students have been studied extensively. But those studies have been done for nursing students. Few number of study findings were indicated that "Examined LS using conceptual models are frequently applied by nursing researchers than the Myers-Briggs type Indicator (MBTI). Meanwhile the studies have contributed significantly to understanding teaching-learning preferences of students (7). Numerous learning theories and assessment tools are available and have been used extensively in the field of LS studies(8).While alternative approaches to learning can be used successfully, it is thought that students will learn more quickly and easily if they are able to utilize their preferred style (9). The value of developing awareness of LS can help students to recognize their strengths, weakness. They work more efficiently when self-directed in learning and They develop effective collaborative relationships with others $(10,11)$.

A Learning style(LS) is an individual student's chosen, preferred, or characteristic approach to learning 12,13) Educational researchers, such as $(14), 15,16)$ and Vermunt (1996) 17) have developed models of LS. According to (18), a LS can be considered a developmental and biological characteristic that individuals use to concentrate, process, internalize, and retain new and difficult information. Psychologist and educational theorist David Kolb developed a four-stage learning cycle designed to describe how learning by experience takes place. This experiential learning cycle contains four different phases: Concrete Experience (CE), Reflective Observation (RO), Abstract Conceptualization (AC), and Active Experimentation (AE). According to Kolb, we can begin at any point in this cycle. Learning, he suggests, is essentially a process that involves looping around and around this cycle. It is the four phases of this cycle that serve as the basis for Kolb's learning styles. While LS are often criticized as overly simplistic or lacking in empirical research, Kolb's model remains one of the most popular today (19). Each of the four LS is characterized by preferences in two areas of the learning cycle. For example, people with assimilating LS prefer to learn though AC and RO. In other words, they like to think about abstract ideas and combine these thoughts with their own observations. The accommodating LS, on the other hand, is characterized by preferences for CE and AE. These learners like to gain hands-on-experience and then experiment with different methods and ideas. Assimilators tend to be watchers, while accommodators tend to be doers (19).

\subsection{Kolb's-Matrix View}

It is often easier to see the construction of Kolb's LS in terms of a two-by-two matrix. The diagram also highlights Kolb's terminology for the four LS; diverging, assimilating, and converging, accommodating:

Table 1. Kolb's LSS - matrix view.

\begin{tabular}{lll}
\hline & Doing (-AE) & Watching(-RO) \\
\hline Feeling & Accommodating (CE/AE) & Diverging (CE/RO) \\
Thinking & Converging (AC/AE) & Assimilating (AC/RO) \\
\hline
\end{tabular}

Thus, for example, a person with a dominant LS of 'doing' rather than 'watching' the task, and 'feeling' rather than 'thinking' about the experience, will have a LS which combines and represents those processes, namely an 'accommodating' LS, in Kolb's terminology.

\subsection{The Experiential Learning Cycle}

The Kolb's experiential LS theory is typically represented by a four stage learning cycle in which the learner's touches all the bases:

- Concrete Experience (a new experience of situation is encountered, or a re-interpretation of existing experience).

- Reflective Observation (of the new experience. of particular importance are any inconsistencies between experience and understanding).

- Abstract Conceptualization (reflection gives rise to a new idea, or a modification of an existing abstract concept).

- Active Experimentation (the learner applies them to the world around them to see what results)(McLeod, 2010a).

Effective learning is seen when a person progresses through a cycle of four stages: of (1) having a CE followed by (2) observation of and reflection on that experience which leads to (3) the formation of abstract concepts (analysis) and generalizations (conclusions) which are then (4) used to test hypothesis in future situations, resulting in new experiences.

Self-Directed Learning (SDL) has been one of the predominant issues in the study and practice of medical education in the last 4 decades. The impetus for the growing trend of SDL, in undergraduate as well as postgraduate education results from the rapid advancement of science (22). SDL, as outlined by Knowles (1975), clearly assigns the major responsibility for learning to the student. Within the framework provided by the goals and objectives of the program, students should be able to determine their own learning goals, how best to achieve their objectives, how to select learning resources, and how to measure their own progress. SDL is a component of life-long learning, which is now a clear expectation for all occupational therapists as practitioners within a regulated health profession. SDL provides students with the essential skills needed to work within changing practice contexts (23))SDL readiness scale was developed, field-tested and revised by Guglielmino in 1977. It has since been translated into French, Spanish (Castilian, Cuban, and Colombian), Japanese, Chinese, Korean, German, Finnish, Greek, Portuguese, Italian, Farsi, 
Malay, Dutch, Polish, Russian, Afrikaans, Latvian, Lithuanian, and Turkish, and used in hundreds of research efforts in 40 countries, including a large number of master's theses and doctoral dissertations. The Fisher-self-directed learning Readiness Scale (Fisher-SDLRS) is cited in numerous articles and books relating to adult education, and is generally recognized as the most valid and widely-used instrument of its kind $(23,24,25)$.

\section{Concrete \\ Experience (doing / having an experience)}
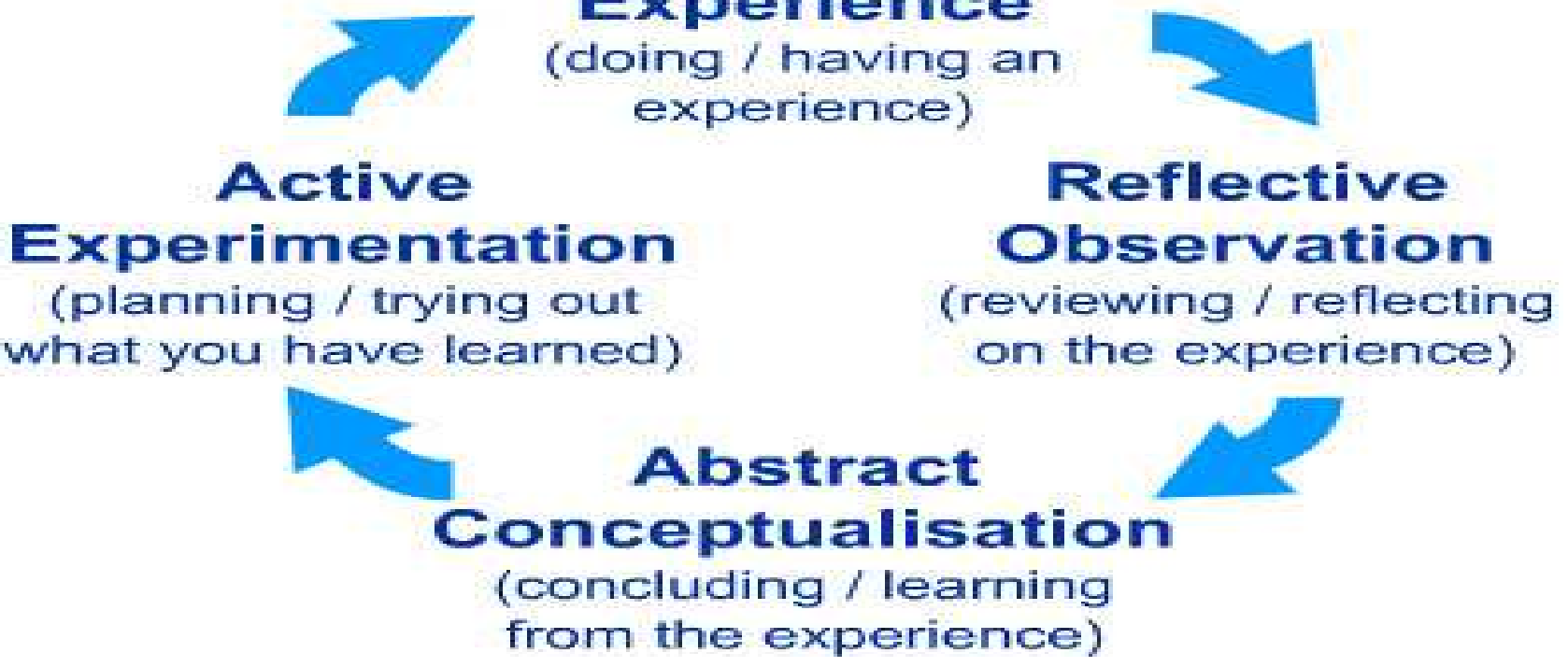

Source:-http://www.ldu.leeds.ac.uk/ldu/sddu_multimedia/kolb/static_version.php

Figure 1. The Experiential Learning Cycle.

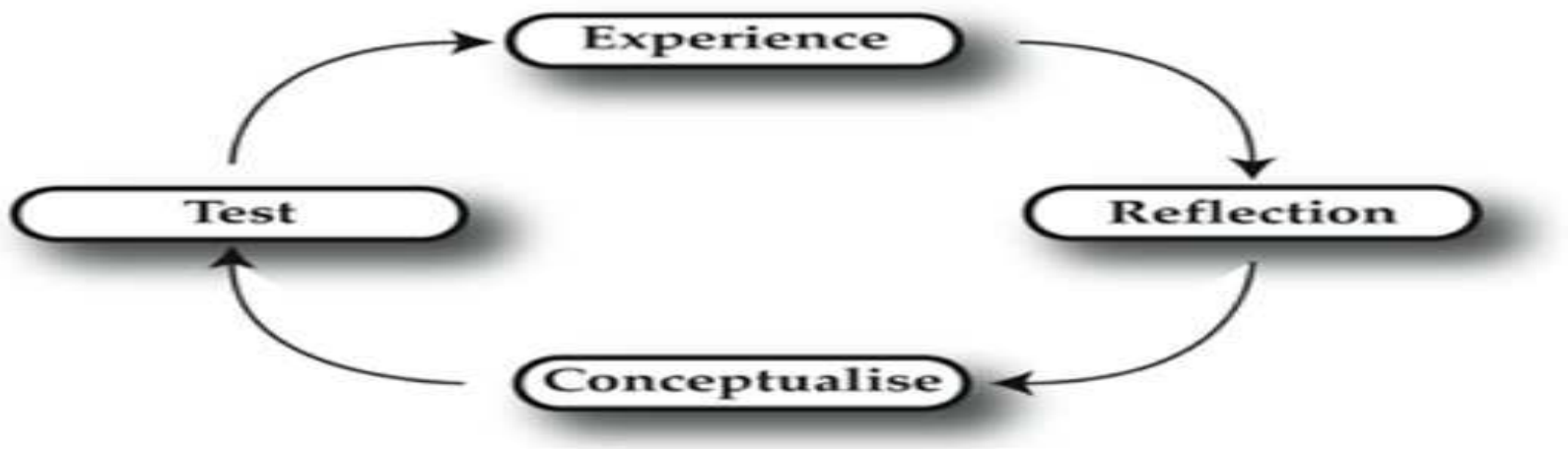

McLeod, S. A.(2010).Kolb-Learning Styles. Retrieved from http://www.simplypsychology.org/learning-kolb.html

Figure 2. Effective learning stages cycle.

\section{Methods and Materials}

\subsection{Study Area and Period}

This study was conducted in the school of nursing and midwifery, TUMS, Tehran, Iran. The study was conducted from March, 2013 to February, 2014.

\subsection{Study Design}

A correlational cross-sectional study was conducted to determine the relationship between LS, SD in Learning and undergraduate nursing students' academic achievement in TUMS, Tehran, Iran.

\subsection{Study Population}

All undergraduate nursing students who attended at the school of nursing and midwifery and they can fulfill the criteria for inclusion were enrolled in the study. Each undergraduate nursing student who attended at the school of nursing and midwifery that can fulfill the criteria for inclusion was enrolled in the study. Both Not any work experience as a teacher among the participants, the participants should be native Persian speakers and Age $\geq 17$ years were sample characteristics for this study.

\subsection{Sample Size}

The sample size was determined by employing stratified 
population in order to determine statistical formula. Formula the size was determined. The study was included at least 221 students, then the total students who enrolled at study area is 743 nursing students(Tehran University Of Medical Sciences, 2012). The required minimum sample is obtained and with 5\% non-response rate of total were calculated 232 were obtained.

\subsection{Sampling Techniques}

All undergraduate students from the total number of students about 232 were selected. The method of sampling Quota Stratified Sampling Techniques (QSST).Total undergraduate nursing students in the school from 2009-2013 were about 743 persons (Refer to Tasble.2). Regarding the sampling technique, study group from the source of population were choices for each academic year of the nursing study (year 1-4). The number of samples through quota stratified sampling techniques were 67 from year one, 69,65 , and 31 from second year, third year and fourth year on academic year of their education respectively.

\subsection{Instruments and Measurements}

The questionnaires were self-administered and consists of: Socio-demographic characteristics which includes: Age, Sex, Marital Status, Permanent residence, Living area, Department, grade level, academic achievement, Average hours of independent study, Average hours for social activities, and students study planning. It was consists all these variables and each variable have their own options. Learning styles questionnaires: On Kolb's LS in nursing student's questionnaire. For LS (LS) was used the Persian Version of the Kolb's LSI and SDL in nursing students' was used the Persian Version of the SDLR in Nursing students which were showed valid and reliable instruments. The Kolb's LSI questionnaire consists of 12 -items with 1-"least like you", 2-"Third most like you",3-"Second most like you", and 4-"Most like you" grading option. It requires the respondents to rank order, their preferences. It classifies an individual's LSs based on 4 major kinds of capability. The inventory measures an individual's relative emphasis on 4 learning modes or scales: $\mathrm{CE}, \mathrm{RO}, \mathrm{AC}$ and $\mathrm{AE}$ (Kolb, 2010).Academic Achievement Academic Achievement was measured using students' final grades for the courses taught by the case method (Teheran University of Medical Science International Campus, 2013). We were used the scale of 20 for academic achievement of nursing students. A measurement of individual grade of scaling from" Excellent" to "good" to "acceptable " to "weak'. It is classified scales;18-20 which indicates "Excellent", 16-18 indicates "Great", 13-16 indicates "Good", 10-13 indicates "Acceptable' and 0-10 indicates "weak" of students' academic achievement.

Self-Directed Learning Readiness for undergraduate nursing student: On their aspect of self-directed learning. The self -directed learning too is a 40-items five-point scale listing a number of self-directed learning skills in nursing students. The students were required to rate themselves in terms of the degree to which they believed the statement in the scale described their approach to learning. It is desirable while mentioning here that researcher was compared to Fisher as follows "Completely Disagree", for those who were not self-directed and the range of scores is less than 40 . A measurement of individual sense of ranging from "Completely agree "to "Strongly Disagree". The instrument is a 40-items five-point linker scale .Scores was assigned as $<48.75$ "Strongly Disagree" for Self-management, $>48.75$ "Strongly agree" for Self-management, $<45$ "Strongly Disagree" for Self -Desired in Learning, $>45$ "Strongly agree" for self-desired in learning, $<56.25$ "Strongly Disagree" for Self-control, $>56.25$ Strongly agree" for Self-control and $<150$ "Strongly Disagree" for Total SD in Learning, $>150$ "Strongly agree" for Total SD in Learning $(26,27$.$) .$

\subsection{Data Collection Procedure}

Data was collected from students through an anonymous self-administrated questionnaire. The questionnaire was divided into four sections including (a) demographic profile,(b) Kolb's Learning Style Inventory,(c) Fisher's SelfDirected in Learning Readiness questionnaire,(d) academic achievement. An initial version of the questionnaire was piloted on a small group of nursing students as the context was validated by a panel of expertise in nursing education. All ethical considerations were applied in this study. The time which allowed filling all questionnaires for one participant was 30 minute but they could take more time.

\subsection{Data Processing and Analysis}

The SPSS version 21, software program was used to analyze the data. The data was analyzed using descriptive measures of central tendency including means and standard deviations to determine the range and differences between the scores. The Pearson product moment correlation provided information on the relationship between LS, self-directed in learning and undergraduate nursing students' academic achievement. Chi-square and T-tests was used to employ to determine variations in academic achievement among nursing students. The level of significance for the study was considered $\mathrm{p}<0.05$. The Inferential statistics was calculated using all the participant scores as well as course subgroup

\subsection{Operational Definition}

Learning style: LS refer to performance way of information processing as determined by LSI. In this study, LSI describes the way student learn and how student deal with ideas and day to day situations in their life and the instrument which consists of 12 sentences with 4 option or prioritized ways (Kolb, 1984a). Grading the option for each sentence according to how well you think each one fits with how you would go about learning something. In addition, the grading scale: 1-indicates least like you; 2-indicates third most like you; 3-indicates second most like you and 4indicates most like you. A measurement of individual sense of ranging from "least like you" to "third most like you" to 
"second most like you" to "Most like you". The instrument is a 12-items five-point linker scale. Scores was assigned as 112 for "least like you", 13-24 for "third most like you", 25-36 for "second most like you", 37-48 for "Most like you" LS in nursing students (Sani et al., 2012).

Academic achievement: The final GPA obtained from courses taught. In this research, it was used the scale of 20 for academic achievement of nursing students. A measurement of individual grade of scaling from "Excellent" to "good" to "acceptable " to "weak'. It is classified scales; 18-20 which indicates "Excellent", 16-18 indicates "Great", 13-16 indicates "Good", 10-13 indicates "Acceptable" and 010 indicates "weak" of students' academic achievement

\subsection{Data Quality Control}

To ensure the quality of data, first the questionnaire was pretested. The pretest was conducted in 55 of the participants at randomly selected undergraduate student's ways from the study setting. Training was given for the data collectors and supervisors before the actual data collection. Every day after data collection, questionnaires were reviewed and checked for completeness, accuracy and clarity by the supervisors and principal investigators.

\subsection{Ethical Consideration}

Ethical approval of the research proposal was obtained from the ethical review committee of Tehran University of Medical Sciences, School of Nursing and Midwifery, Department of Nursing. The letter directed to the Tehran University of Medicals Sciences, School of Nursing and Midwifery Dean, and department heads. Permission was sought from the Medical Research Council of Tehran, Iran and the Joint Research Ethics Committee, Ethical Review Boards for the protection of human subjects in the study. Clear communication was conducted with education office and departments and programs was arranged to conduct the study. Furthermore after brief explanation of the purpose of study, written consent was obtained from the study participants and those voluntary to participate was provided the questionnaire to fill. The confidentiality was assured by excluding their name and not participate or withdraw at any point from the study was respected.

\section{Result}

\subsection{Demographic Characteristics}

The result shows that the majority of participants $(60.8 \%$; $\mathrm{n}=141$ ) were at age group between 20-25 years old The finding in figure 2, indicates that almost more than half of participants $(61.6 \% ; n=143)$ were female. Majority of participants $(80.4 \% ; n=205)$ were single. $98.3 \%(n=228)$ were living in metropolitan areas. Shows that more than half of participants $(53 \% ; n=123)$ have lived with their families. As it was shows in Table 2, the majority of subjects in this study $(31 \% ; n=72)$ were in the second acdemic year. More than half of subjects $(66.4 \%)$ had independent study for less than two hours a day. reveals that the majority of students (28\%) had the social activities between 2-4 hours a day. most of subjects (44\%) had self-study planning on the exam weeks.

Table 2. Frequency Distribution on Socio-demographic characteristics of undergraduate Nursing students in Tehran University of Medical Sciences, School of Nursing and Midwifery, Tehran, Iran, 2014.

\begin{tabular}{|c|c|c|c|c|}
\hline Roll No & Variables & Classification & Frequency & Percentage \\
\hline \multirow{3}{*}{1} & \multirow{3}{*}{ Sex } & Female & 143 & 62 \\
\hline & & Male & 89 & 38 \\
\hline & & Total & 232 & 100 \\
\hline \multirow{4}{*}{2} & \multirow{4}{*}{ Age } & $<20$ & 78 & 34 \\
\hline & & $20-25$ & 141 & 60 \\
\hline & & $>20$ & 13 & 6 \\
\hline & & Total & 232 & 100 \\
\hline \multirow{3}{*}{3} & \multirow{3}{*}{$\begin{array}{l}\text { Marital } \\
\text { Status }\end{array}$} & Single & 205 & 88 \\
\hline & & Married & 27 & 12 \\
\hline & & Total & 232 & 100 \\
\hline \multirow{3}{*}{4} & \multirow{3}{*}{$\begin{array}{l}\text { Permanent } \\
\text { Residence }\end{array}$} & City & 228 & 98 \\
\hline & & Village & 4 & 2 \\
\hline & & Total & 232 & 100 \\
\hline \multirow{5}{*}{5} & \multirow{5}{*}{$\begin{array}{l}\text { Living } \\
\text { place }\end{array}$} & Dormitory & 77 & 33 \\
\hline & & Personal Home & 29 & 13 \\
\hline & & Family home & 123 & 53 \\
\hline & & Rent & 3 & 1 \\
\hline & & Total & 232 & 100 \\
\hline \multirow{5}{*}{6} & \multirow{5}{*}{$\begin{array}{l}\text { Academic } \\
\text { year }\end{array}$} & First year & 64 & 27.6 \\
\hline & & Second year & 72 & 31 \\
\hline & & Third year & 65 & 28 \\
\hline & & Fourth Year & 31 & 13.4 \\
\hline & & Total & 231 & 100 \\
\hline \multirow{5}{*}{7} & \multirow{5}{*}{$\begin{array}{l}\text { Average } \\
\text { hour of } \\
\text { independent } \\
\text { study }\end{array}$} & $0-2$ & 154 & 66.4 \\
\hline & & $2-4$ & 64 & 27.6 \\
\hline & & $4-6$ & 13 & 5.6 \\
\hline & & $6-8$ & 1 & 0.4 \\
\hline & & $10+$ & 232 & 100 \\
\hline \multirow{7}{*}{8} & \multirow{7}{*}{$\begin{array}{l}\text { Average } \\
\text { hours of } \\
\text { social } \\
\text { activities }\end{array}$} & $0-2$ & 57 & 24.6 \\
\hline & & $2-4$ & 65 & 28 \\
\hline & & $4-6$ & 62 & 26.7 \\
\hline & & $6-8$ & 19 & 8.2 \\
\hline & & $8-10$ & 12 & 5.2 \\
\hline & & $10+$ & 17 & 7.3 \\
\hline & & Total & 232 & 100 \\
\hline \multirow{4}{*}{9} & \multirow{4}{*}{$\begin{array}{l}\text { Self study } \\
\text { planning }\end{array}$} & Regularly & 76 & 32.5 \\
\hline & & Exam weeks & 102 & 44 \\
\hline & & $\begin{array}{l}\text { Before the day } \\
\text { of exam }\end{array}$ & 54 & 23.3 \\
\hline & & Total & 232 & 100 \\
\hline
\end{tabular}

The result in this study shows that students in this study had mainly AC learning styles. Although female students had a higher AC mean score than male students $(32.36 \pm 6.408$; $31.02 \pm 5.717$ respectively) but there was no statistically relationship between LS and Gender. In addition, students in age between 20-25 years old had a higher AC mean score than other groups. There was no statistically relationship between learning style and age classification, academic level. However, there was relationship between AC learning style 
and academic achievement $(\mathrm{F}=4.890 ; \mathrm{P}=0.003)$ and $\mathrm{AE}$

(Table.3)

learning style and academic achievement as component

Table 3. Students'learning styles according to some of demographic characteristics.

\begin{tabular}{|c|c|c|c|c|c|c|c|}
\hline \multirow[b]{2}{*}{ Variables } & \multirow[b]{2}{*}{ Description } & \multicolumn{2}{|c|}{ Concrete Experience } & \multicolumn{2}{|l|}{ Reflective Observation } & \multicolumn{2}{|l|}{ Total } \\
\hline & & $\mathbf{M} \pm \mathbf{S D}$ & $\begin{array}{l}\mathbf{F} \\
\mathbf{P}\end{array}$ & $\mathbf{M} \pm \mathbf{S D}$ & $\begin{array}{l}\mathbf{F} \\
\mathbf{P}\end{array}$ & $\mathbf{M} \pm \mathbf{S D}$ & $\begin{array}{l}\mathbf{F} \\
\mathbf{P}\end{array}$ \\
\hline \multirow{3}{*}{ Sex } & Female & $27.86 \pm 4.848$ & \multirow{3}{*}{$\begin{array}{l}\mathrm{F}=2.482 \\
\mathrm{P}=0.117\end{array}$} & $32.36 \pm 6.408$ & \multirow{3}{*}{$\begin{array}{l}\mathrm{F}=2.57 \\
\mathrm{P}=0.110\end{array}$} & $120.13 \pm 0.906$ & \multirow{3}{*}{$\begin{array}{l}\mathrm{F}=0.648 \\
\mathrm{P}=0.422\end{array}$} \\
\hline & Male & $28.90 \pm 4.941$ & & $31.02 \pm 5.717$ & & $120.03 \pm 0.923$ & \\
\hline & Total & $28.26 \pm 4.899$ & & $31.84 \pm 6.174$ & & $120.09 \pm 0.911$ & \\
\hline \multirow{4}{*}{ Age(Year) } & $<20$ & $27.59 \pm 4.96$ & \multirow{4}{*}{$\begin{array}{l}\mathrm{F}=1.265 \\
\mathrm{P}=0.284\end{array}$} & $29.06 \pm 5.317$ & \multirow{4}{*}{$\begin{array}{l}\mathrm{F}=0.233 \\
\mathrm{P}=0.792\end{array}$} & $119.99 \pm 0.781$ & \multirow{4}{*}{$\begin{array}{l}F=1.034 \\
P=0.357\end{array}$} \\
\hline & $20-25$ & $28.67 \pm 4.992$ & & $29.58 \pm 5.655$ & & $120.13 \pm 0.781$ & \\
\hline & $>25$ & $27.85 \pm 2.940$ & & $29.69 \pm 5.893$ & & $120.31 \pm 0.855$ & \\
\hline & Total & $28.26 \pm 4.899$ & & $29.41 \pm 5.557$ & & $120.09 \pm 0.911$ & \\
\hline \multirow{5}{*}{ Academic year } & First year & $29.03 \pm 5.102$ & \multirow{5}{*}{$\begin{array}{l}\mathrm{F}=1.012 \\
\mathrm{P}=0.388\end{array}$} & $29.64 \pm 5.022$ & \multirow{5}{*}{$\begin{array}{l}\mathrm{F}=0.928 \\
\mathrm{P}=0.428\end{array}$} & $119.97 \pm 0.942$ & \multirow{5}{*}{$\begin{array}{l}\mathrm{F}=0.80 \\
\mathrm{P}=0.493\end{array}$} \\
\hline & Second year & $28.36 \pm 4.567$ & & $29.11 \pm 5.479$ & & $120.11 \pm 0.848$ & \\
\hline & Third year & $27.63 \pm 4.712$ & & $28.88 \pm 5.920$ & & $120.22 \pm 0.944$ & \\
\hline & Fourth Year & $27.74 \pm 5.568$ & & $30.77 \pm 6.009$ & & $120.06 \pm 0.929$ & \\
\hline & Total & $28.26 \pm 4.899$ & & $2941 \pm 5.557$ & & $120.09 \pm 0.911$ & \\
\hline
\end{tabular}

Table 3. Continue.

\begin{tabular}{|c|c|c|c|c|c|c|c|}
\hline \multirow[b]{2}{*}{ Variables } & \multirow[b]{2}{*}{ Description } & \multicolumn{2}{|c|}{ Abstract Conceptualization } & \multicolumn{2}{|c|}{ Active Experimentation } & \multicolumn{2}{|l|}{ Total } \\
\hline & & $\mathbf{M} \pm \mathbf{S D}$ & $\begin{array}{l}\mathbf{F} \\
\mathbf{P}\end{array}$ & $\mathbf{M} \pm \mathbf{S D}$ & $\begin{array}{l}\mathbf{F} \\
\mathbf{P}\end{array}$ & $\mathbf{M} \pm \mathbf{S D}$ & $\begin{array}{l}\mathbf{F} \\
\mathbf{P}\end{array}$ \\
\hline \multirow{3}{*}{ Sex } & Female & $29.35 \pm 6.102$ & \multirow{3}{*}{$\begin{array}{l}\mathrm{F}=0.049 \\
\mathrm{P}=0.824\end{array}$} & $30.57 \pm 6.292$ & \multirow{3}{*}{$\begin{array}{l}\mathrm{F}=0.001 \\
\mathrm{P}=0.972\end{array}$} & $120.13 \pm 0.906$ & \multirow{3}{*}{$\begin{array}{l}\mathrm{F}=0.648 \\
\mathrm{P}=0.422\end{array}$} \\
\hline & Male & $29.52 \pm 4.578$ & & $30.60 \pm 5.900$ & & $120.03 \pm 0.923$ & \\
\hline & Total & $29.41 \pm 5.55$ & & $30.58 \pm 6.132$ & & $120.09 \pm 0.911$ & \\
\hline \multirow{4}{*}{ Age(Year) } & $<20$ & $31.76 \pm 5.955$ & \multirow{4}{*}{$\begin{array}{l}\mathrm{F}=0.112 \\
\mathrm{P}=0.894\end{array}$} & $31.58 \pm 5.863$ & & $119.99 \pm 0.781$ & \multirow{4}{*}{$\begin{array}{l}\mathrm{F}=1.034 \\
\mathrm{P}=0.357\end{array}$} \\
\hline & $20-25$ & $31.96 \pm 6.231$ & & $29.93 \pm 6.306$ & $\mathrm{~F}=2.029$ & $120.13 \pm 0.781$ & \\
\hline & $>25$ & $31.15 \pm 7.244$ & & $31.62 \pm 5.157$ & $\mathrm{P}=0.134$ & $120.31 \pm 0.855$ & \\
\hline & Total & $31.84 \pm 6.174$ & & $30.58 \pm 6.132$ & & $120.09 \pm 0.911$ & \\
\hline \multirow{5}{*}{ Academic year } & Firstyear & $31.47 \pm 5.754$ & \multirow{5}{*}{$\begin{array}{l}\mathrm{F}=4.890 \\
\mathrm{P}=0.003\end{array}$} & $29.83 \pm 6.202$ & & $119.97 \pm 0.942$ & \multirow{5}{*}{$\begin{array}{l}\mathrm{F}=0.804 \\
\mathrm{P}=0.493\end{array}$} \\
\hline & Second year & $30.42 \pm 5.535$ & & $32.22 \pm 6.271$ & & $120.11 \pm 0.848$ & \\
\hline & Third year & $34.18 \pm 6.151$ & & $29.52 \pm 5.829$ & $\begin{array}{l}F=2.748 \\
P=0.044\end{array}$ & $120.22 \pm 0.944$ & \\
\hline & FourthYear & $31.03 \pm 7.273$ & & $30.52 \pm 5.750$ & & $120.06 \pm 0.929$ & \\
\hline & Total & $31.84 \pm 6.174$ & & $30.58 \pm 6.132$ & & $120.09 \pm 0.911$ & \\
\hline
\end{tabular}

Table 4. Students' Academic achievement according to some of socio-demographics characteristics.

\begin{tabular}{|c|c|c|c|c|c|c|c|c|}
\hline \multirow{3}{*}{ Variables } & \multirow{3}{*}{ Classification } & \multicolumn{7}{|c|}{ Academic Achievement } \\
\hline & & \multicolumn{2}{|c|}{ 10-13 (Acceptable) } & \multicolumn{2}{|c|}{ 13-16 (good) } & \multicolumn{2}{|c|}{ Total } & \multirow{2}{*}{ Result } \\
\hline & & $\mathbf{n}$ & $\%$ & $\mathbf{n}$ & $\%$ & $\mathbf{n}$ & $\%$ & \\
\hline \multirow{3}{*}{ Sex } & Female & 1 & 7.7 & 57 & 47.9 & 138 & 61.3 & \multirow{3}{*}{$\begin{array}{l}\text { Fisher exact test }=50.988 \\
\mathrm{df}=1 \text {, } \\
\mathrm{P}=0.000\end{array}$} \\
\hline & Male & 12 & 92.3 & 62 & 52.1 & 87 & 87 & \\
\hline & Total & 13 & 100 & 119 & 100 & 225 & 225 & \\
\hline \multirow{3}{*}{ Marital Status } & Single & 10 & 76.9 & 102 & 85.7 & 199 & 88.4 & \multirow{3}{*}{$\begin{array}{l}\text { Fisher exact test }=8.125 \\
\mathrm{df}=1 \\
\mathrm{P}=0.032\end{array}$} \\
\hline & Married & 3 & 23.1 & 17 & 14.3 & 26 & 11.6 & \\
\hline & Total & 13 & 100 & 119 & 100 & 225 & 100 & \\
\hline \multirow{4}{*}{ Self-study planning } & Regularly & 2 & 15.4 & 33 & 27.7 & 70 & 31.1 & \multirow{4}{*}{$\begin{array}{l}\text { Fisher's exact test }=9.558 \\
d f=1 \\
P=0.103\end{array}$} \\
\hline & Exam weeks & 6 & 46.2 & 52 & 43.7 & 101 & 44.9 & \\
\hline & Before the day of exam & 5 & 38.5 & 34 & 28.6 & 54 & 24 & \\
\hline & Total & 13 & 100 & 119 & 100 & 225 & 100 & \\
\hline \multirow{3}{*}{ Permanent Residence } & City & 13 & 100 & 116 & 97.5 & 221 & 98.2 & \multirow{3}{*}{$\begin{array}{l}\text { Fisher's exact test }=1.982 \\
d f=1 \\
P=0.730\end{array}$} \\
\hline & Village & 0 & 0 & 3 & 2.5 & 4 & 1.8 & \\
\hline & Total & 13 & 100 & 119 & 100 & 225 & 100 & \\
\hline
\end{tabular}




\begin{tabular}{|c|c|c|c|c|c|c|c|c|}
\hline \multirow{3}{*}{ Variables } & \multirow{3}{*}{ Classification } & \multicolumn{7}{|c|}{ Academic Achievement } \\
\hline & & \multicolumn{2}{|c|}{ 10-13 (Acceptable) } & \multicolumn{2}{|c|}{ 13-16 (good) } & \multicolumn{2}{|c|}{ Total } & \multirow{2}{*}{ Result } \\
\hline & & $\mathbf{n}$ & $\%$ & $\mathbf{n}$ & $\%$ & $\mathbf{n}$ & $\%$ & \\
\hline \multirow{5}{*}{ Living place } & Living Dormitory & 4 & 30.8 & 42 & 35.3 & 77 & 34.2 & \multirow{5}{*}{$\begin{array}{l}\text { Fisher's exact } \\
\text { test }=7.192 \\
\mathrm{df}=1 \\
\mathrm{P}=0.691\end{array}$} \\
\hline & Home & 1 & 7.7 & 18 & 15.1 & 28 & 12.4 & \\
\hline & Family Home & 7 & 53.8 & 58 & 48.7 & 117 & 52.0 & \\
\hline & Rental & 1 & 7.7 & 1 & 0.8 & 3 & 13 & \\
\hline & Total & 13 & 100 & 119 & 100 & 225 & 100 & \\
\hline \multirow{5}{*}{$\begin{array}{l}\text { Average hours of } \\
\text { independent study }\end{array}$} & $0-2$ & 11 & 84.6 & 86 & 72.3 & 150 & 66.7 & \multirow{5}{*}{$\begin{array}{l}\text { Fisher's exact } \\
\text { test }=13.994 \\
d f=1 \\
P=0.158\end{array}$} \\
\hline & $2.1-4$ & 2 & 15.4 & 27 & 22.7 & 63 & 28 & \\
\hline & $4.1-5$ & 0 & 0.0 & 5 & 4.2 & 11 & 4.9 & \\
\hline & $6-8$ & 0 & 0.0 & 1 & 0.8 & 1 & 0.4 & \\
\hline & Total & 13 & 100 & 119 & 100 & 225 & 100 & \\
\hline \multirow{5}{*}{$\begin{array}{l}\text { Academic Year/level of } \\
\text { study }\end{array}$} & First-year & 1 & 7.7 & 31 & 26.1 & 57 & 25.3 & \multirow{5}{*}{$\begin{array}{l}\text { Fisher's exact } \\
\text { test }=15.332 \\
d \mathrm{~d}=1 \\
\mathrm{P}=0.041\end{array}$} \\
\hline & Second Year & 6 & 46.2 & 44 & 37 & 72 & 32 & \\
\hline & Third-year & 6 & 46.2 & 26 & 21 & 65 & 28.9 & \\
\hline & Fourth-Year & 0 & 0 & 18 & 15.1 & 31 & 13.8 & \\
\hline & Total & 13 & 100 & 119 & 100 & 225 & 100 & \\
\hline
\end{tabular}

Table 4. Continue.

\begin{tabular}{|c|c|c|c|c|c|c|c|c|}
\hline \multirow{3}{*}{ Variables } & \multirow{3}{*}{ Classification } & \multicolumn{7}{|c|}{ Academic Achievement } \\
\hline & & \multicolumn{2}{|c|}{ 16-18 (Great) } & \multicolumn{2}{|c|}{ 18-20(Excellent) } & \multicolumn{2}{|c|}{ Total } & \multirow{2}{*}{ Result } \\
\hline & & $\mathbf{n}$ & $\%$ & $\mathbf{n}$ & $\%$ & $\mathbf{n}$ & $\%$ & \\
\hline \multirow{3}{*}{ Sex } & Female & 77 & 55.6 & 3 & 100 & 138 & 61.3 & \multirow{3}{*}{$\begin{array}{l}\text { Fisher exact test }=50.988 \\
d f=1, \\
P=0.000\end{array}$} \\
\hline & Male & 13 & 14.4 & 0 & 0 & 87 & 87 & \\
\hline & Total & 90 & 100 & 3 & 100 & 225 & 225 & \\
\hline \multirow{3}{*}{ Marital Status } & Single & 85 & 94.4 & 2 & 66.7 & 199 & 88.4 & \multirow{3}{*}{$\begin{array}{l}\text { Fisher exact test }=8.125 \\
d f=1 \\
P=0.032\end{array}$} \\
\hline & Married & 5 & 5.6 & 1 & 33.3 & 26 & 11.6 & \\
\hline & Total & 90 & 100 & 3 & 100 & 225 & 100 & \\
\hline \multirow{4}{*}{ Self-study planning } & Regularly & 35 & 38.9 & 0 & 0 & 70 & 31.1 & \multirow{4}{*}{$\begin{array}{l}\text { Fisher's exact test }=9.558 \\
d f=1 \\
P=0.103\end{array}$} \\
\hline & Exam weeks & 41 & 45.6 & 2 & 66.7 & 101 & 44.9 & \\
\hline & Before the day of exam & 14 & 15.6 & 1 & 33.3 & 54 & 24 & \\
\hline & Total & 90 & 100 & 3 & 100 & 225 & 100 & \\
\hline \multirow{3}{*}{ Permanent Residence } & City & 89 & 98.9 & 3 & 100 & 221 & 98.2 & \multirow{3}{*}{$\begin{array}{l}\text { Fisher's exact test }=1.982 \\
d f=1 \\
P=0.730\end{array}$} \\
\hline & Village & 1 & 1.1 & 0 & 0 & 4 & 1.8 & \\
\hline & Total & 90 & 100 & 3 & 100 & 225 & 100 & \\
\hline \multirow{5}{*}{ Living place } & Living Dormitory & 30 & 33.3 & 1 & 33.3 & 77 & 34.2 & \multirow{5}{*}{$\begin{array}{l}\text { Fisher's exact } \\
\text { test }=7.192 \\
d f=1 \\
P=0.691\end{array}$} \\
\hline & Home & 9 & 10 & 0 & 0.0 & 28 & 12.4 & \\
\hline & Family Home & 50 & 55.6 & 2 & 66.7 & 117 & 52.0 & \\
\hline & Rental & 1 & 1.1 & 0 & 0 & 3 & 13 & \\
\hline & Total & 90 & 100 & 3 & 100 & 225 & 100 & \\
\hline \multirow{5}{*}{$\begin{array}{l}\text { Average hours of independent } \\
\text { study }\end{array}$} & $0-2$ & 50 & 55.6 & 3 & 100 & 150 & 66.7 & \multirow{5}{*}{$\begin{array}{l}\text { Fisher's exact } \\
\text { test }=13.994 \\
d f=1 \\
P=0.158\end{array}$} \\
\hline & $2.1-4$ & 34 & 37.8 & 0 & 0.0 & 63 & 28 & \\
\hline & $4.1-5$ & 6 & 67 & 0 & 0.0 & 11 & 4.9 & \\
\hline & $6-8$ & 0 & 0.0 & 0 & 0.0 & 1 & 0.4 & \\
\hline & Total & 90 & 100 & 3 & 100 & 225 & 100 & \\
\hline \multirow{5}{*}{ Academic Year/level of study } & First-year & 25 & 27.8 & 0 & 0 & 57 & 25.3 & \multirow{5}{*}{$\begin{array}{l}\text { Fisher's exact } \\
\text { test }=15.332 \\
d f=1 \\
P=0.041\end{array}$} \\
\hline & Second Year & 22 & 24.4 & 0 & 0 & 72 & 32 & \\
\hline & Third -year & 30 & 33.3 & 3 & 100 & 65 & 28.9 & \\
\hline & Fourth-Year & 13 & 14.4 & 0 & 0 & 31 & 13.8 & \\
\hline & Total & 90 & 100 & 3 & 100 & 225 & 100 & \\
\hline
\end{tabular}

Table 4 reveals that undergraduate nursing students in this study had scored good in their academic study. In addition, female students were a high scorer than male students respectively $(55.6 \%$ and $14.4 \%)$. There was a relationship between LS and gender $(\mathrm{P}<0.05)$. There was a relationship between LS and marital status $(\mathrm{P}=0.032)$. There was no relationship between LS and self study, permanent residence, living, place and the average hours of independent study. There was a relationship between LS and academic level $(\mathrm{P}=0.041)$.

This table 5. Reveals that the majority of subjects (57.9\%) were female who have higher level of SDLR than male. 
However, the proportion of high SDLR did not show any students. significant variation with all sociodemographics of the

Table 5. Students' Self-directed learning according to some of socio-demographics characteristics.

\begin{tabular}{|c|c|c|c|c|c|c|c|c|}
\hline \multirow{3}{*}{ Variables } & \multirow{3}{*}{ Classification } & \multicolumn{7}{|c|}{ Self-directed learning } \\
\hline & & \multicolumn{2}{|c|}{$<150$} & \multicolumn{2}{|l|}{$>150$} & \multicolumn{2}{|c|}{ Total } & \multirow{2}{*}{ Result } \\
\hline & & $\mathbf{n}$ & $\%$ & 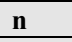 & $\%$ & $\mathrm{n}$ & $\%$ & \\
\hline \multirow{3}{*}{ Sex } & Female & 75 & 64.7 & 66 & 57.9 & 141 & 61.3 & \multirow{3}{*}{$\begin{array}{l}\mathrm{X}^{2}=1.108^{*} \\
\mathrm{df}=1 \\
\mathrm{P}=0.344\end{array}$} \\
\hline & Male & 41 & 35.3 & 48 & 42.1 & 89 & 38.7 & \\
\hline & Total & 116 & 100 & 114 & 100 & 230 & 100 & \\
\hline \multirow{3}{*}{ Marital Status } & Single & 100 & 86.2 & 103 & 90.4 & 203 & 88.3 & \multirow{3}{*}{$\begin{array}{l}\mathrm{x}^{2}=0.953^{*} \\
\mathrm{df}=5 \\
\mathrm{P}=0414\end{array}$} \\
\hline & Married & 16 & 13.8 & 11 & 9.6 & 27 & 11.7 & \\
\hline & Total & 116 & 100 & 114 & 100 & 230 & 100 & \\
\hline \multirow{4}{*}{ Self-study planning } & Regularly & 38 & 32.8 & 38 & 33.3 & 76 & 33 & \multirow{4}{*}{$\begin{array}{l}\mathrm{X}^{2}=0.011^{*} \\
\mathrm{df}=2 \\
\mathrm{P}=1.000\end{array}$} \\
\hline & Exam weeks & 51 & 44 & 50 & 43.9 & 101 & 43.9 & \\
\hline & Before the day of exam & 27 & 23.3 & 26 & 22.8 & 53 & 23 & \\
\hline & Total & 116 & 100 & 114 & 100 & 230 & 100 & \\
\hline \multirow{5}{*}{ Living place } & Living in TUMS Dormitory & 33 & 28.4 & 44 & 38.6 & 77 & 33.5 & \multirow{5}{*}{$\begin{array}{l}\text { Fisher's exact } \\
\text { test }=2.929 \\
\mathrm{df}=1 \\
\mathrm{P}=0.434\end{array}$} \\
\hline & Home & 15 & 12.9 & 13 & 11.4 & 28 & 12.2 & \\
\hline & Family Home & 66 & 56.9 & 56 & 49.1 & 122 & 53.0 & \\
\hline & Rental & 2 & 1.7 & 1 & 0.9 & 3 & 1.3 & \\
\hline & Total & 116 & 100 & 114 & 100 & 230 & 100 & \\
\hline \multirow{5}{*}{$\begin{array}{l}\text { Average hours of } \\
\text { independent study }\end{array}$} & $0-2$ & 80 & 69.0 & 73 & 64.0 & 153 & 66.5 & \multirow{5}{*}{$\begin{array}{l}\text { Fisher's exact } \\
\text { test }=1.746 \\
\mathrm{df}=1 \\
\mathrm{P}=0.709\end{array}$} \\
\hline & $2.1-4$ & 29 & 25 & 34 & 29.8 & 63 & 27.4 & \\
\hline & $4-6$ & 7 & 6 & 6 & 5.3 & 13 & 5.7 & \\
\hline & $6-8$ & 0 & 0.0 & 1 & 0.9 & 1 & 0.4 & \\
\hline & Total & 116 & 100 & 114 & 100 & 230 & 100 & \\
\hline \multirow{7}{*}{$\begin{array}{l}\text { Average hours for social } \\
\text { activities }\end{array}$} & $0-2$ & 34 & 29.3 & 23 & 20.2 & 57 & 24.8 & \multirow{7}{*}{$\begin{array}{l}x^{2}=4.786^{*} \\
d f=5 \\
P=0.449\end{array}$} \\
\hline & $2.1-4$ & 29 & 25 & 25 & 30.4 & 64 & 27.8 & \\
\hline & $4-6$ & 33 & 28.4 & 28 & 24.6 & 61 & 26.5 & \\
\hline & $6-8$ & 7 & 6.0 & 12 & 10.5 & 19 & 8.3 & \\
\hline & $8-10$ & 5 & 4.3 & 7 & 6.1 & 12 & 5.2 & \\
\hline & $10+$ & 8 & 6.9 & 9 & 7.9 & 17 & 7.4 & \\
\hline & Total & 116 & 100 & 114 & 100 & 230 & 100 & \\
\hline \multirow{5}{*}{$\begin{array}{l}\text { Academic Year/level of } \\
\text { study }\end{array}$} & First-year & 34 & 29.3 & 30 & 26.3 & 64 & 27.8 & \multirow{5}{*}{$\begin{array}{l}\text { Pearson } \\
\left(X^{2}\right)=5.018 \\
d f=3 \\
P=0.171\end{array}$} \\
\hline & Second Year & 41 & 35.3 & 31 & 27.2 & 72 & 31.3 & \\
\hline & Third-year & 31 & 26.7 & 33 & 28.9 & 64 & 27.8 & \\
\hline & Fourth-Year & 10 & 8.6 & 20 & 17.5 & 30 & 13 & \\
\hline & Total & 116 & 100 & 114 & 100 & 230 & 100 & \\
\hline
\end{tabular}

The result of F-test indicates (table 7) that there was no significant relationship between LS and Academic Achievement $(\mathrm{P}>0.05)$. The result also indicated that the highest mean score of participants, who are mainly using $\mathrm{AC}$ learning Style, had great score (table.6).

Table 6. Relationship between students' learning styles and academic achievement.

\begin{tabular}{|c|c|c|c|c|c|c|}
\hline \multirow[b]{2}{*}{ Learning Style } & \multicolumn{5}{|c|}{ Academic Achievement } & \multirow[t]{2}{*}{ F-test } \\
\hline & Acceptable $10-13$ & Good 13-16 & Great $16-18$ & Excellent $18-20$ & Total & \\
\hline & Mean \pm S.D & Mean \pm S.D & Mean \pm S.D & Mean \pm S.D & Mean \pm S.D & \\
\hline Concrete experience & $28.62 \pm 4.646$ & $28.69 \pm 5.038$ & $28.10 \pm 4.606$ & $24.33 \pm 3.786$ & $28.39 \pm 4.836$ & $\begin{array}{l}\mathrm{F}=0.972 \\
\mathrm{P}=0.407\end{array}$ \\
\hline Abstract conceptualization & $31.15 \pm 3.602$ & $30.99 \pm 6.146$ & $33.16 \pm 6.363$ & $34.00 \pm 2.646$ & $31.91 \pm 6.157$ & $\begin{aligned} \mathrm{F} & =2.332 \\
\mathrm{P} & =0.075\end{aligned}$ \\
\hline Reflective observation & $30.31 \pm 3.728$ & $30.06 \pm 5.513$ & $28.40 \pm 5.842$ & $28.67 \pm 5.508$ & $29.39 \pm 5.592$ & $\begin{array}{l}\mathrm{F}=1.655 \\
\mathrm{P}=0.178\end{array}$ \\
\hline Active Experimentation & $30.46 \pm 7.827$ & $30.34 \pm 5.615$ & $30.44 \pm 6.512$ & $33.00 \pm 4.359$ & $30.43 \pm 6.083$ & $\begin{array}{l}\mathrm{F}=0.185 \\
\mathrm{P}=0.907\end{array}$ \\
\hline Total & $120.54 \pm 1.127$ & $120.08 \pm 1.038$ & $120.10 \pm 0.654$ & $120.00 \pm 0.000$ & $120.12 \pm 0.904$ & $\begin{array}{l}\mathrm{F}=1.022 \\
\mathrm{P}=0.387\end{array}$ \\
\hline
\end{tabular}

Table 7 reveals that there was not a statistically significant correlation between LS and SDL. The result also showed that the mean score of self-management, desired for learning,
Self-control and the overall SDLR were similar in a student's $(31.84 \pm 6.174 ; 31.83 \pm 6.177)$ who had using AC of LS. However, there was a relationship between self management 
and $\mathrm{AC}$ of $\mathrm{LS}(\mathrm{F}=8.485 ; \mathrm{P}=0.004)$.

Table 7. Relationship between students' learning styles and self-directed learning.

\begin{tabular}{|c|c|c|c|c|c|c|c|c|c|c|}
\hline \multirow{2}{*}{ SDL } & \multicolumn{2}{|c|}{ Concrete Experience } & \multicolumn{2}{|c|}{ Reflective Observation } & \multicolumn{2}{|c|}{$\begin{array}{l}\text { Abstract } \\
\text { Conceptualization }\end{array}$} & \multicolumn{2}{|c|}{ Active Experimentation } & \multicolumn{2}{|l|}{ Total } \\
\hline & $\mathbf{M} \pm \mathbf{S D}$ & $\begin{array}{l}\mathbf{F} \\
\mathbf{P}\end{array}$ & $\mathbf{M} \pm \mathbf{S D}$ & $\begin{array}{l}\mathbf{F} \\
\mathbf{P}\end{array}$ & $\mathbf{M} \pm \mathbf{S D}$ & $\begin{array}{l}\mathbf{F} \\
\mathbf{P}\end{array}$ & $\mathbf{M} \pm \mathbf{S D}$ & $\begin{array}{l}\mathbf{F} \\
\mathbf{P}\end{array}$ & $\mathbf{M} \pm \mathbf{S D}$ & $\begin{array}{l}\mathbf{F} \\
\mathbf{P}\end{array}$ \\
\hline Self-Management & $\begin{array}{l}28.26 \pm 4 \\
899\end{array}$ & $\begin{array}{l}\mathrm{F}=0.377 \\
\mathrm{P}=0.540\end{array}$ & $\begin{array}{l}29.41 \pm 5 \\
557\end{array}$ & $\begin{array}{l}\mathrm{F}=2.631 \\
\mathrm{P}=0.106\end{array}$ & $\begin{array}{l}31.84 \pm \\
6.174\end{array}$ & $\begin{array}{l}\mathrm{F}=8.485 \\
\mathrm{P}=0.004\end{array}$ & $30.58 \pm 6.132$ & $\begin{array}{l}\mathrm{F}=2.147 \\
\mathrm{P}=0.144\end{array}$ & $\begin{array}{l}120.09 \\
\pm 0.911\end{array}$ & $\begin{array}{l}\mathrm{F}=0.000 \\
\mathrm{P}=0.999\end{array}$ \\
\hline $\begin{array}{l}\text { Self-designed in } \\
\text { learning }\end{array}$ & $\begin{array}{l}28.26 \pm 4 \\
899\end{array}$ & $\begin{array}{l}F=0.029 \\
P=0.865\end{array}$ & $\begin{array}{l}30.58 \pm 6 \\
132\end{array}$ & $\begin{array}{l}F=1.162 \\
P=0.282\end{array}$ & $\begin{array}{l}31.84 \pm \\
6.174\end{array}$ & $\begin{array}{l}F=0.228 \\
P=0.634\end{array}$ & $29.41 \pm 5.557$ & $\begin{array}{l}\mathrm{F}=0.334 \\
\mathrm{P}=0.564\end{array}$ & $\begin{array}{l}120.09 \\
\pm 0.911\end{array}$ & $\begin{array}{l}\mathrm{F}=0.122 \\
\mathrm{P}=0.727\end{array}$ \\
\hline Self-Control & $\begin{array}{l}28.30 \pm 4 \\
900\end{array}$ & $\begin{array}{l}F=0.028 \\
P=0.868\end{array}$ & $\begin{array}{l}29.37 \pm 5 \\
557\end{array}$ & $\begin{array}{l}F=1.342 \\
P=0.248\end{array}$ & $\begin{array}{l}31.83 \pm \\
6.177\end{array}$ & $\begin{array}{l}\mathrm{F}=1.193 \\
\mathrm{P}=0.276\end{array}$ & $30.59 \pm 6.154$ & $\begin{array}{l}\mathrm{F}=0.052 \\
\mathrm{P}=0.819\end{array}$ & $\begin{array}{l}120.10 \\
\pm 0.915\end{array}$ & $\begin{array}{l}\mathrm{F}=0.095 \\
\mathrm{P}=0.758\end{array}$ \\
\hline Total & $\begin{array}{l}28.30 \pm 4 \\
900\end{array}$ & $\begin{array}{l}F=0.000 \\
P=0.983\end{array}$ & $\begin{array}{l}29.37 \pm 5 \\
557\end{array}$ & $\begin{array}{l}F=2.751 \\
P=0.099\end{array}$ & $\begin{array}{l}31.83 \pm \\
6.177\end{array}$ & $\begin{array}{l}F=1.720 \\
P=0.191\end{array}$ & $30.59 \pm 6.154$ & $\begin{array}{l}\mathrm{F}=0.000 \\
\mathrm{P}=0.993\end{array}$ & $\begin{array}{l}120.10 \\
\pm 0.915\end{array}$ & $\begin{array}{l}\mathrm{F}=1.298 \\
\mathrm{P}=0.256\end{array}$ \\
\hline
\end{tabular}

\section{Discussion}

The present study also determined a relationship between learning styles and a number of sociodemographic characteristics. Undergraduate nursing students are some of the most studied groups with regard to learning styles and Kolbs LSI is the most frequently used instrument, which determine LS. No study assessing LS of undergraduate nursing has used the most recent version of Kolb LSI (Version 3.1). But, this instrument was currently used in the United State of America to identify LS Inventory(28).This study indicates that students in this study had mainly AC learning styles. Although female students had a higher AC mean score than male students. However, there was no relationship between LS and Gender. In contrast, D'Amore et al (2012) showed that female students had a higher reflective observation (RO) score than male students(29). This study result also indicated that there was no statistically relationship between learning style and age classification, academic level. However, there was relationship between AC learning style and academic achievement and $\mathrm{AE}$ learning style and academic achievement as a component. In similar, Smith (2012) showed that there was no relationship between LS and age, previous employment or nursing experience(30). This is unexpected since it would be anticipated that those with more experience would have more a balanced LS. This is agreed with Salehi (2007) who found the relationship between academic years and $\mathrm{AC}$ was found statistically significant as well as between academic years and AE (31). This study result indicated that there was no statistically significant difference according to total LS among students in different sex $(\mathrm{P}>0.05)$. This is agreed with Salehi (2007) who observed that there was no significant relationship was found between the LS and gender (31). In contrast, the study which was conducted by Fleming et al (2011) who found the most common dominant LS in first year was the dual learning category $(35 \%)$ while a large proportion of the students $(53 \%)$ in their final year had no dominant LS (32). The preferred LS of students in their first (69\%) and final (57\%) year was reflector. In comparison with a previous study which conducted by Salehi (2007) who found the nursing students' preference in LS, senior students were less likely to use $\mathrm{AE}$ than Junior undergraduate nursing students. However, senior undergraduate nursing students were more likely compared with all other academic years/level of study who use AC to $\mathrm{AE}$ (31).A more recent study of undergraduate nursing students found that students had a high preference for the reflector LS followed by the theorists LS $(33,34)$. In contrast, the study was conducted by Erol (2010) in Turkey, who explored the Kolb LSs inventory was used to explore the LS of the study group(35).This is agreed with Aziz et al (2013), who determined Reflector the LS was the most common among the students .The preferred LSs were statistically independent of the demo graphic variables examined such as level of academic years, sex, race and pre-University qualifications $(36,37)$.Similarly, in the current study we show that first-year nursing and nursing used the AC learning style closely followed by the $\mathrm{AE}$ in learning style. LS were significantly different at the two time points and there was a significant relationship between some LSs and students' age but not with academic achievement $(32,38)$. Failure of find significant difference between the LS and socio demographic characteristics of nursing students was not surprising because it conforms to the reviewed literature. Because they represent same population. The main reasons might be: the questionnaire used may not be a suitable tool to detect any gender differences in LS or it may not include questions related to the areas of differences. Students who are RO learning style in the previous studies are motivated themselves to discover the relevancy of an environment or situation. They may like to reason from concrete, specific information and to explore what a system has to offer, and they may prefer to have information presented to them in a detailed, systematic, reasoned manner. This study reveals that the majority of subjects $(57.9 \%)$ were female who have higher level of self directed in learning than male. However, the proportion of high Self directed in learning readiness did not show any significant variation with all sociodemographic of the students. This study result related with the study which was conducted by walker et al (2007) who demonstrated that students enrolled in an accelerated baccalaureate nursing program self -reported statistically greater self-directedness and motivation towards learning than their traditional learning than their traditional counterparts, independent of age (39). This is agreed with ,Kocaman et al (2009) who 
found a total of 59 students (Response rate, 85\%) completed the Self directed in learning readiness scale at five different time points: at the beginning of each academic year and at program completion $(40,41)$. This study result indicated that there was lower SDL redness scale in current study than the previous studies. The reason might be: SDL is the main learning skills for accelerated undergraduate nursing students and the discrepancy is attributed to the increase awareness as a result of the information given through the different methods at class room. Thus the results of this study support the agreement made by Azer (2008) claimed that selfdirected learning is an adult learning process that makes use of feedback to fulfill the detected learning needs(42). This study results compared with a study which was conducted by Williams in Australia (2013) who found a female participants produced higher scores across each subscale and total SDL (43). This supports the work of Williams, who examined of SDL readiness of paramedic undergraduates students on their sex (44) .These results are difference with the previous study done by Miller, 2010, who found the majority of participants spent between two up to six hours a week an independent study (45). The current study reveals that undergraduate nursing students in this study had scored good in their academic study. In addition; female students were a high scorer than male students respectively. In contrast, the study which was conducted by Gemeay (2013) who investigate the self-concept and its relation with the academic achievement among nursing students, $43 \%$ of studied subjects were in third year and regarding academic achievement the subjects had $(14.5 \%)$ either good or excellent. However, in this study finding showed a variety and distribution of academic achievement regarding academic years (46). In this study also indicated that there was a relationship between LS and academic level. This study result indicated that there was slightly differ in differ in academic achievement than previous study which conducted by Gemeay (2013). The reason maybe: the fact that academic achievement was generally higher in female undergraduate nursing students in this study than the previous study. The reason for the number of female two times more than male undergraduate nursing students may be due to related the entrance requirement to school of nursing and midwifery. Further researcher in this academic achievement and gender area is necessary to examine why females do better than males and it appears that more emphasis should be placed on time management skills during their academic year. The present study shows that in a diverse group of undergraduate nursing students, the learning style. The AC learning style was indicated that the main dominant for the present study group of participants. Undergraduate nursing students in the present study are mainly AC learning style in their study. In contrast, Baker et al (2007) and Gyeong and Myung (2008) who found that nursing students have been shown to be concrete learners(47)The probable reason for this difference may be due to the service given on LS becomes increased from time to time in this study area, Students' need of practical uses for ideas and theories and this may suggest that those who learn by AC learning style (thinking) rely more on seeking the best knowledge. The current study revealed that the most frequency learning style of students was AC followed by $\mathrm{AE}$, RO and CE in LS of students. The result which related to the watching types of LS more represented in this study is consistent with the studies conducted by (20, 21,48,49), Which determined nursing students to be a predominant thinking LS. In contrast, regarding to the results of other different researchers, nursing students tend to have either an doing, watching or feeling LSs as revealed in the previous studies. For example, the LS in this study are compared with the study done by Westcott and Johnson (2011) in the Education, Learning, Styles, Individual differences Network (ELSIN) conference at Qatar. The finding which indicated that, the majority or $64 \%$ of faculty who participated had as their strength AC or thinking, analyzing, and planning systematically. The average LS quadrant of faculty was Assimilating AC plus RO to gather information, synthesize it logically, focus on abstract concepts, prefer lectures and models, and think things through. Faculty results contrasted with the averages of the majority of students, especially with new students to the university. The most predominant style according to the Kolb LSI for $50 \%$ of all students was AE or doing, experimenting, taking risks, and influencing others through action. The present study revealed that the majority of undergraduate nursing students $(90.52 \%)$ have high level of SDLR(score $\geq 150$ ). This agree with ,Safavi et al(2010) who stated that majority of Jordanian and Iranian nursing students had high level of SDLR and perceived themselves as independent learner (26). The current study shows there was no significant relationship between learning styles and academic achievement. The highest mean score of participants, who are mainly using AC learning style, had great scored. Similar with present study which was conducted by Panahi et $\mathrm{al}(2012)$, Ahanachian et al(2012) showed that there was no significant relationship between LS and academic achievement(50,51). In contrast, Yang et al (2013) showed that academic achievement of students who were convergers was significantly higher than those who were Divergers/accommodators (52). Different researcher have been used to explain academic achievement besides different in ability, which are not easy to control, students have specific LS that may influence their academic achievement $(53,54,55,56,57,58)$.

\section{Conclusion}

This is the first study which conducted among undergraduate nursing students' relationship between LS, their academic achievement and SDL in TUMS, school of nursing and midwifery, Tehran, Iran. More research with larger groups is needed to generalize this result. Undergraduate nursing students often prefer a AC learning style as a main dominant. This may suggest that those who learn by $\mathrm{AC}$ learning style (thinking) rely more on seeking the best knowledge. Consideration for individual learning 
styles is fundamental in designing effective training program. Furthermore, it is necessary to provide students with the skills to seek analyze and utilize information effectively. SDL may be used to help undergraduate nursing students learning in connection with way of the teacher led. SDLR is indicated as a set of feature that generally becomes more pronounced in this study participants as they get knowledge. This progression to SDL requires a change in emphasis from teaching to facilitation and the views of Undergraduate nursing students regarding to their role in SD in learning requirement to be determined in depth. Self-directed learning is a method of teaching and learning increasingly used in nursing education, and the result of assessing SDLR is useful for educators to select appropriate teaching and learning methods for their students. Therefore, the present study revealed that the overall SDLR of participants was at a high level. In order to implement this method at the Department of medical surgical nursing, it was important to assess SDL readiness of students. It indicates that low scored by current undergraduate students who had participated in this study. Therefore, these undergraduate nursing students in study area, who perceive themselves positively, have a high degree of success. Moreover, the difference may happen due to better education and information of the students at present time and awareness of the students who participated in this study. Considering this achievement of nursing students, it seems necessary to take in to account the qualities education based on their learning style during study. This may students with direction for need of further learning styles. The highest of SDLR and the dominant AC learning style among Undergraduate nursing students will have a positive suggestion for their education and Upgrading nursing education. In nursing, the rapidly changing health care delivery and practices require sound LS, SDL system and decision making skills. Despite the lack of statistical significance in the relationship between self-directed learning and LS in this study, there are several important findings that deserve future research. The variables that were found to have statistical significance should be examined further. These include sex, Age, marital status, CGPA, academic year/level of study. Meanwhile, Practitioners in universities, school of nursing and midwifery, higher education need consideration in identifying the factors that lead to change their LS and academic achievement. Further research is needed to include variables such as Academic achievement, $\mathrm{RO}, \mathrm{AE}$ in LS that may influence self-directed learning in undergraduate nursing students as suggested by different previous studies. Data from this study can be used to further develop SDL in nursing students and to improve teaching and learning methods. Further research should follow the development of nursing students' SDL abilities over their academic years as well as their lifelong careers in Iran. Researchers need to consider more effective means of assessing nursing students' LS and, their academic achievement and SDL so that a higher response rate can be seen and the finding can also be achieved. Replicating the study with only nursing students who have been accepted into a nursing program, might address engagement issues (diverse perspectives, communication with faculty and peers, asking questions in class, class discussions, perceived difficulty of course work, and preparation for class) that are specific to students who are committed to a particular course of study. Although the majority of subjects in this study were admitted into the nursing program, the majority of subjects from the freshman course had not yet been admitted. This study might be designed as a longitudinal study over the Four year period that a student would be in the nursing program.

\section{Authors Contribution}

AA has made substantial contributions to beginning and design, collection of data, analysis and interpretation of data and in drafting the manuscripts and correcting the comment given by the advisors. SG and NM involved in revising the research paper and the manuscript critically with important intellectual context; also participated in the approval of the final version to be published in its design and coordination. He participated in the approval and funding process, participated in the design of the study participated in its design and coordination .In addition, all Authors had greater contribution in reviewing the manuscript English and topography and helped to draft the manuscript.

\section{Acknowledgement}

We are grateful to all persons who provide technical help to this study and assisted in writing the manuscript. We are acknowledged international Campus Head for the material support. We are thankful to all our department technical staffs for their excellent technical support. We are grateful to all the participants for their cooperation and all my best friends and my families for financial support during this successful research. We would like to acknowledge gratefully for Science PG Journal due to their cooperation and funding for this publication

\section{References}

[1] Cleveland State University School of Nursing (2012). Philosophy and conceptual frame work.

[2] Arthurs, J. B(2007). A juggling act in the classroom: managing different learning styles. . teaching and learning in nursing, 2, 2-7.

[3] Dmirbas, O.O., Demirkan, H(2007).Learning styles of design students and the relationship between academic performance and gender in design education. . Learning and Instruction 17, 345-359.

[4] Whitton, $\mathrm{N}(2010)$. Learning with digital games: a practical guide to engaging students in higher education. . new york: routledge.

[5] Sawchuk, $R(2012)$. Learning nurses resources network, 2012.: Learning and Learning style. learning nurse newsletter. 
[6] Nasrabadi, H. B., Mousavi, S., Farsan, Z. K(2012). The contribution of critical thinking attitude and cognitive learning style in predicating academic achievement of medical university students. iranian journal of medical education., 12, 285-296.

[7] Malloy, H. D(2007). The relationship of learning style, academic achievement, and psychological type of baccalaureate nursing students, proquest.

[8] Noguera, J. S., Wageman,J(2011). Spanish efl undergraduate students' perceptions of learning styles. nordic journal of english studies, 10, 77-101.

[9] Brown, t., Cosgriff, T.,French, G(2008). Learning style preferences of occupational therapy, physiotherapy and speech pathology students: a comparative study. internet journal of allied health sciences \& practice, 6,12 .

[10] Provident, I., M.L,L., Dolhi, C.,Jeffcoat, J(2009). Becoming a fieldwork "educator": enhancing your teaching skills. ot practice., 14, suppl: $2 \mathrm{p}$, ce1-8.

[11] Rogers, K. M. A(2009). A preliminary investigation and analysis of student learning style preferences in further and higher education. . Journal of Further and Higher Education, $33,13-21$.

[12] Cassidy, S(2004). learning styles: An overview of theories, models, and measures. educational psychology 24, 419-444.

[13] Kolb and kolbs (2005). Experential learning experience as the source of learning and development.

[14] Kolb, D. A(1984).Experiential learning: Experience as the source of learning and development.

[15] Felder, R. M., Silverman, L. K(1988)"Learning and teaching styles in engineering education. " engr. education, 78, 674681 .

[16] Felder, R M(1993). "Reaching the second tier: learning and teaching styles in college science education," j. college science teaching, 23, 286-290 . an updated presentation of the felder-silverman model.

[17] Dunn, R., Honigsfeeld, A.,Doolan, L.S(2009). Impact of learning style instructional strategies or students achievement $\&$ attitudes;perceptions of educations of educators institutions. education journal, 82, 135-140.

[18] Honigsfeld, a. \& dunn, r. 2009. learning-style responsive approaches for teaching typically performing and at-risk adolescents. clearing"house:. "a"journal"of"educational"strategies," issues"and"ideas, 82, 220-224.

[19] Cherry, K.(2013). A closer look at kolb's four stytle of learning .about .com guide.

[20] Mcleod, S.A (2010). Kolb's learning styles and experiential learning cycle simply psychology.

[21] Mcleod, S.A (2010). Kolb's learning styles and experiential learning cycle - simply psychology. .

[22] Ainoda, N., Onishi, H., Yasuda, Y(2005). Definitions and goals of "self-directed learning"in contemporary medical education literature. Ann Acad Med Singapore, 34, 515-9.

[23] Mcmaster University (2008). Educational frame work. school of nursing and rehabilitation science.
[24] Merriam, S. ,Caffarella, R.(eds.)(2007). Learning in adulthood: a comprehensive guide ( 3 rd ed.). .

[25] Merriam, S., Caffarella, R., Baumgartner, 1. (eds.) 2007. learning in adulthood: a comprehensive guide (4th ed.).

[26] Safavi, M., Scooshtari, Z., S.H, Mahamoodi, M., Yarmohammadian, M (2010). Self-directed learning readiness and learning styles among nursing students of isfahan university of medical sciences. iranina journal of medical education, 10, 27-35.

[27] Safavi, M., Z.S.H, S., Mahmoodi, M., Yarmohammadian, M( 2010). Self-directed learning styles among nursing students of isfahan university of medical scieneces. iranian journal of medical education., 10, 27-35.

[28] Laeeq, K., Weatherly, R. A., Carrott, A., Pandian, V., Cummings, C. W.,Bahatti, N. I. (2009). learning styles in two otolarngology residency programs the laryngoscope, 119, 2360-2365.

[29] D'amore, A., James, S.,Maitchell, E. K.L(2012).Learning styles of first -year undergraduate nursing and midwifery students: a cross-sectional survey utilizing the kolb learning style inventory. nurse education today, 32, 506-515.

[30] Smith, A(2010). Learning styles of registreted nurses enrolled in an online nursing program journal of professional nursing, $26,49-53$

[31] Salehi, S( 2007). Nursing students' preferred learning style. journal of medical education., 11, 1-5.

[32] Fleming , S., Mckee ,G.,Huntley-Moore ,S( 2011). Undergraduate nursing students' learning styles: a longitudinal study. nurse educ today. , 31, 444-9. doi: 10.1016/j.nedt.2010.08.005.

[33] Rassool, G. H., Rawaf, S(2007). Learning style preferences of undergraduate nursing students. nurs stand, 21, 35-41.

[34] Rassool , G. H., Rawaf , S(2008). The influence of learning styles preference of undergraduate nursing students on educational outcomes in substance use education. nurse educ pract., 8, 306-14. doi: 10.1016/j.nepr.2008.02.001. epub 2008 mar 25 .

[35] Erol Gurpinar, E., Alimoglu, M. K., Mamakli, S., Aktekin, M( 2010). Can learning style predict student satisfaction with different instruction methods and academic achievement in medical education? Adv Physiol Educ 35, 307-311, 2011;doi:10.1152/advan.00047.2011.

[36] Tahernezhad, E., Behjat, F., Kargar, AA(2014).The Relationsh ip between Language Learning Anxiety and Language Learning Motivation among Iranian Intermediate EFL Learners. International Journal of Language and Linguistics. Special Issue: Innovations in Foreign Language Teaching. Vol. 2 ( 6-1): 35-48. doi: 10.11648/j.ij1l.s.2014020601.16

[37] Aziz, Z., Tey, X. Y., Alwi, S., Jet, C. N( 2013). Learning style prefrences of pharmacy students. the european journal of social and behavioural sciences, eiss:2301-2218, 820-837.

[38] Fleming , S., Mckee , G.,Huntley-Moore , S(2011). undergraduate nursing students' learning styles: a longitudinal study. nurse educ today, 31, 444-9. doi: 10.1016/j.nedt.2010.08.005. epub 2010 sep 21. 
[39] Walker, J. T., Martin, T M., Haynie, L., Norwood, A., White, J.,Grant, L(2007). Preferences for teaching methods in a baccalaureate nursing program: how second degree and traditional students differ. . nursing education perspectives, 28 , 246-250.

[40] Aghaei, H., Behjat, F., Rostampour, M (2014). Investigating the Relationship between Iranian High School Female Students' Spiritual Intelligence, Language Proficiency and Self-Esteem. International Journal of Language and Linguistics. Special Issue: Innovations in Foreign Language Teaching 2 (6-1): 19-27. doi: 10.11648/j.ij1l.s.2014020601.14

[41] Kocaman , G., Dicle , A., Ugur, A(2009). A longitudinal analysis of the self-directed learning readiness level of nursing students enrolled in a problem-based curriculum. $\mathrm{j}$ nurs educ, 48, 286-90.

[42] Azer, S. A(2008). Navigating problem-based learning. london: . elsevier.

[43] Williams, B., Boyle, M., Winship,C., Brightwell, R., Devenish, S., munro, g. 2013b. examination of self-directed learning readiness ofparamedic undergraduates: a multi-institutional study. journal of nursing education and practice, 3, 102-111. doi: $10.5430 /$ jnep.v3n2p102.

[44] Williams, B., Boyl, M., Winship, C., Brightwen, R., Devenish, S., Graham, M(2013). Examination of self-directed learning readiness of paramedic undergraduates a multi-institutional study. . journal of nursing education and practice.3 (2):102111., 3, 102-111.

[45] Miller, M. P(2010). Are first year undergraduate students nurses prepared for self-directed learning? nursing times.net.

[46] Gemeay, E., Behilak, S., Kanona, E.,Mansour, E. A(2013). Self -concept and academic achievements among nursing students. life sci j, 10, 1466-1470.

[47] Baker, C. M., Pesult, D. J., Mcdaniel, A. M.,Fisher, M. L(2007). Evaluating the impact of problem-based learning on learning styles of master's students in nursing administration. journal of professional nursing 23, 214-219.
[48] Kazu, I. Y(2009).The effect of learning styles on education and the teaching process. . journal of social sciences, 5 (1), 117. , 5, 1-17.

[49] Marchese, T.J(2012). " The new conversations about learning. insights from neuroscience and anthropology, . . cognitive science and workplace studies, 1-15.

[50] Panahi, R., Kazemi, S.,Rezaie, A(2012). The relationship of learning styles to academic achievement: the role of gender and academic discipline. . developmental psychology: iranian psychologists., 8, 189-196.

[51] Ahanchian, M., Mohamadzadeghasr , A., Garavand , H. ,Hosseini, A(2012). Prevalent learning styles among nursing and midwifery students and its association with functionality of thinking styles and academic achievement; a study in mashhad school of nursing and midwifery. iranian journal of medical education., 12 577-588.

[52] Yang, S., Ha , E., Lee , O., Sim, I., Park, Y., Nam, H., Kim , $\mathrm{J}(2012)$. Academic achievement, self-directed learning, and critical thinking disposition according to learning styles of nursing students. journal of korean academy of fundamentals of nursing, 19, 334 .

[53] Deary, I. J., Strand, S., Smith, P.,Fernandes,C(2007). Intelligence and educational achievement. intelligence,. 35, 13-21.

[54] Erdogan, Y., Bayram, S., Deniz, L(2008). Factors that influence academic achievement and attitudes in web based education,. international journal of instruction, 1, 31-48.

[55] Ferla, J., Valcke, M., Cai, Y(2009). Academic self-efficacy and academic self-concept: reconsidering structural relationships. . learning and individual differences, 19, 499505 .

[56] Holliday, A(2009). Understanding the implications self concept and academic self - concept has on african.

[57] O'sullivan, E. M(2009). The demographic and academic profile of irish dental school faculty members. Journal of the Irish Dental Association, 55, 296-301. 